\title{
IMPROVEMENT IN HIPPOCAMPAL KINDLING ANALYSIS THROUGH COMPUTATIONAL PROCESSING DATA
}

\author{
Joacir Graciolli Cordeiro 1,2, Alberto Capurro ${ }^{3,4}$, Ad Aertsen ${ }^{3,4}$, \\ Karina Kohn Cordeiro ${ }^{1,2}$, João Cândido Araújo ${ }^{2}$, Andreas Schulze-Bonhage ${ }^{1,3}$
}

\begin{abstract}
The kindling phenomenon is classically investigated in epileptology research. The present study aims to provide further information about hippocampal kindling through computational processing data. Adult Wistar rats were implanted with dorsal hippocampal and frontal neocortical electrodes to perform the experiment. The processing data was obtained using the Spike2 and Matlab softwares. An inverse relationship between the number of "wet dog shakes" and the Racine's motor stages development was found. Moreover it was observed a significant increase in the afterdischarge (AD) duration and its frequency content. The highest frequencies were, however, only reached at the beginning of behavioral seizures. During the primary AD, fast transients (ripples) were registered in both hippocampi superimposed to slower waves. This experiment highlights the usefulness of computational processing applied to animal models of temporal lobe epilepsy and supports a relevant role of the high frequency discharges in temporal epileptogenesis.
\end{abstract}

KEY WORDS: hippocampal kindling, temporal epilepsy, experimental model.

\begin{abstract}
Aprimorando a análise do modelo de kindling hipocampal com o auxilio de processamento computacional
Resumo - O fenômeno de kindling é classicamente utilizado no campo da epileptologia experimental. Este trabalho objetiva aprofundar a análise do modelo kindling hipocampal através de processamento computacional. Ratos wistar adultos receberam eletrodos hipocampais dorsais e neocorticais frontais para a realização do experimento. $O$ processamento dos dados encontrados foi realizado pelos softwares Matlab e Spike2. Encontrou-se uma relação inversa entre wet dog shakes e o desenvolvimento dos estágios motores de Racine. A duração e o conteúdo de freqüência das pós-descargas hipocampais aumentaram durante o processo, sendo observadas descargas de alta freqüência (ripples) em ambos os hipocampos durante as pós-descargas primárias, superimpostas a ondas lentas. As mais altas freqüências, entretanto, foram apenas atingidas com o início das crises epilépticas. A utilização de sistemas computacionais para a confecção e análise do modelo de epilepsia temporal é ressaltada e reforça-se a relevância do papel das altas freqüências na epileptogênese temporal.
\end{abstract}

PALAVRAS-CHAVE: kindling hipocampal, epilepsia temporal, modelo animal.

Temporal lobe epilepsy (TLE) is the most frequent epilepsy form in adults' and its major cause is mesial temporal sclerosis (MTS), which comprises around $60-70 \%$ of TLE cases ${ }^{2}$. MTS presents with a high clinical refractoriness rate, medical treatment being able to provide seizure control in only $20-40 \%$ of the cases ${ }^{1,3,4}$. In refractory patients surgical treatment may be indicated, with the current rate of favorable outcome with microsurgery reach- ing around 67 to $85 \%$ of the patients ${ }^{4,5}$. Despite the different treatment modalities, a significant amount of patients remain with poor epilepsy control. In order to improve the current MTS treatment, it is important to try to achieve a better understanding of temporal lobe epileptogenesis. To fulfill this purpose, development of animal models of temporal lobe epilepsy have become a valuable tool. The method of hippocampal kindling is high-

Albert-Ludwigs-University, Freiburg, Germany: 'Center for Epilepsy, Dept. Neurosurgery, University Medical Center Freiburg, Albert-Ludwigs-University, Freiburg, Germany; ${ }^{2}$ Department of Neurosurgery, Hospital de Clínicas, Federal University of Paraná, Curitiba PR, Brazil; ${ }^{3}$ Bernstein Center for Computational Neuroscience Freiburg, Albert-Ludwigs-University, Freiburg, Germany; ${ }^{4}$ Neurobiology and Biophysics, Faculty of Biology, Albert-LudwigsUniversity, Freiburg, Germany. Supported by BMBF- Germany (01GQ0420, C3).

Received 11 December 2008, received in final form 14 May 2009. Accepted 17 June 2009.

Dr. Joacir Graciolli Cordeiro - Breisacherstr. 64 - PLZ 79106 Freiburg im Breisgau - Germany. E-mail: joacirgc@hotmail.com 
lighted over other animal models due to its behavioral and electrophysiological features. Kindling is know as the phenomenon in which ictal discharges and epileptic behavioral manifestations progressively appear and increase gradually in complexity, thus favoring the investigation of seizure generation ${ }^{6,7}$. Advances in computational field may also contribute to epileptological studies. The Spike2 software (Science Products GmbH, Hofheim, Germany) allows the synchronization between the electrophysiological recording and the animal behavioral pattern. It also permits the association with other softwares to sophisticate local field potentials (LFP) signal analysis. An example is the coupling with the Matlab software (The MathWorks, Natick, USA), which integrates the signal processing form to the construction of spectrograms, which facilitates the signal evaluation. The objective of this study is to evaluate the use of computational analytical tools applied to the LFP signal recorded from rats submitted to the hippocampal kindling model.

\section{METHOD}

In order to perform the experiment, 12 adult Wistar rats of both sexes (non-pregnant), with weight between 250 and 350 grams were used. The animals were obtained through the Uniklinik Freiburg (Germany) and their use was approved by its ethical commission in compliance with the regulation for experimental animal models. The animals were individually housed during all the experiment period in transparent cages alternating, 12 hours in daylight and 12 hours in darkness. All experiments were held at the same time during the day light cycle. Food and water intake was ad libitum. Three rats were excluded due to technical problems with the electrodes. Anesthesia for cerebral electrode implantation was performed with intraperitoneal injection of ketamine $10 \%$ (100 mg/kg), xylosine $2 \%(3 \mathrm{mg} / \mathrm{kg}$ ) and atropine $0.5 \mathrm{mg} / \mathrm{ml}(0.1 \mathrm{mg} / \mathrm{kg})$. For post-operative analgesia, subcutaneous buprenorfine $(0.05 \mathrm{mg} / \mathrm{kg})$ was used. The animals were placed in the stereotactic frame and burr holes were drilled in the skull according to the coordinates for the dorsal hippocampus ${ }^{8}$. A bipolar twisted platinum electrode was implanted $(125 \mu \mathrm{m}$ diameter and mean impedance of $413 \pm 220 \mathrm{k} \Omega$ at $10 \mathrm{~Hz})$ on the right dorsal hippocampus $(\mathrm{Y}=-3.6 ; \mathrm{X}=-2.4 ; \mathrm{Z}=$ -3.5). A tungstenium monopolar electrode was also implanted ( $60 \mu \mathrm{m}$ diameter and mean impedance of $1220 \pm 544 \mathrm{k} \Omega$ at $10 \mathrm{~Hz}$ ) on both dorsal hippocampi anteriorly and posteriorly to the platinum electrode $(Y=-2.6 ; X= \pm 1.5 ; Z=-3.2$ and $Y=-4.6 ; X= \pm 3.5$; $Z=-3.4$ ). Additionally, two monopolar tungstenium electrodes were implanted in both frontal neocortices aiming at the primary motor area (1 $\mathrm{mm}$ anterior to the coronal suture), one monopolar reference electrode above the left cerebellum and one occipital screw for grounding. Fixation to the skull was performed with dental cement and cranial screws and the electrodes were plugged into the multiple channel connector.

A multiple channel connector was plugged into the MPA8I pre- amplifier, which was linked to the PGA32 signal amplifier. PGA32 was coupled to the analogue-digital converter CED Power1401 and the generated signal was displayed by the Spike2 software. The behavioral pattern was registered and synchronized to the LFP signal and exhibited in real time. One week after the implantation, hippocampal kindling was started. A daily electric stimulus was applied (for five consecutive days separated by two days interval). Biphasic square waves at $60 \mathrm{~Hz}$ with $0.4 \mathrm{~ms}$ of pulse width, $\pm 0.5 \mathrm{~mA}$ of amplitude were applied during 1.6s. The LFP signal was filtered from $1 \mathrm{~Hz}$ to $5 \mathrm{kHz}$, recorded at a sampling rate of $10.4 \mathrm{kHz}$ and 500 times amplified. The obtained behavioral response was classified according to the Racine and Pinel and Rover seizure scales ${ }^{9,10}$ (Table 1).

Sessions were individually repeated until each rat reached ten consecutive behavioral seizures with a minimum Racine score of five (i.e. fully kindled state). After process completion, the animals underwent intracardiac perfusion under deep anesthesia for histological verification of electrode position. Recorded LFP were analyzed in order to determinate the number of wet dog shakes (WDS) and the primary after discharge (1AD) duration. WDS are a stereotyped motor patter (mimicking the shake of a wet dog), which are associated to a characteristic electroencephalographic discharge. WDS quantification was done by

Table 1. Behavioral seizure classification.

\begin{tabular}{lcl}
\hline & Stage & Behaviour \\
\hline Racine & 1 & Oroalimentary movements \\
behavioral scale & 2 & Head nodding \\
& 3 & Anterior limb clonus \\
& 4 & Dorsal extension (rearing) \\
& 5 & Loss of balance and falling \\
Pinel and Rovner & 6 & Repeated falling \\
behavioral scale & 7 & Violent jumping and running \\
& 8 & Stage 7 with tonic period \\
\hline
\end{tabular}

Source: References 9 and 10. Pinel and Rovner behavioral scale adds three posterior stages to the Racine scale.

Table 2. Kindling process subdivision.

\begin{tabular}{ll} 
D0 & first session \\
D1 & first quarter \\
D2 & second quarter \\
D3 & third quarter \\
D4 & fourth quarter (first generalized behavioral seizure) \\
D5 & fifth generalized behavioral seizure \\
D6 & fully kindled state \\
\hline
\end{tabular}

Arbitrary subdivision, in which the first kindling session of each rat was named DO. The individual number of session needed to reach the first generalized behavioral seizure was divided into 4 quarters (D1 to D4). The session in which the rat presented the fifth generalized behavioral seizure was named D5. The fully kindled state was named D6 (the $10^{\text {th }}$ consecutive Racine 5 seizure). 


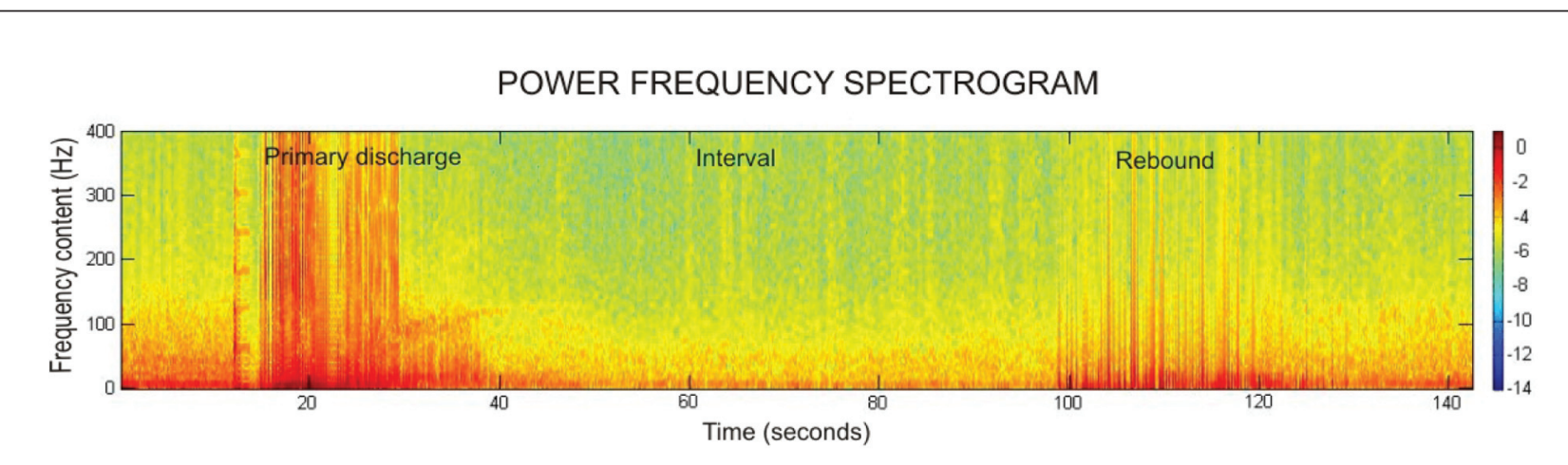

Fig 1. AD power frequency spectrogram. Representative power frequency spectrogram processed from the LFP signal recorded from a kindling session. In the frequency spectrogram a clearer differentiation and analysis of the three typical periods of a hippocampal AD (primary AD, interval and rebound) was observed. Scale bar in $\log _{10}\left[\mathrm{mV}^{2}\right]$. Source: Experiment data.

Table 3. Behavioral seizure duration.

\begin{tabular}{cccccccccccc}
\hline Phase & Rat1 & Rat2 & Rat3 & Rat4 & Rat5 & Rat6 & Rat7 & Rat8 & Rat9 & Mean & SD \\
\hline D4 & 25.8 & 30 & 25 & 32 & 6.4 & 11.7 & 14.2 & 8.8 & 31.4 & 20.6 & 10.3 \\
D5 & 29.3 & 31.8 & 38.4 & 33.2 & 14.7 & 9.1 & 14 & 26.5 & 23 & 24.4 & 10 \\
D6 & 36 & 37.2 & 34.9 & 28.9 & - & 18.9 & 28.3 & 34.1 & 37.2 & 32.2 & 5.9 \\
\hline
\end{tabular}

As the process reached advanced phases the behavioral seizure duration increased, being maximal with the fully kindled state (D6). A statistically significant difference between D4 and D6 ( $<<0.05)$ was observed. Values in seconds; SD: standard deviation.

direct observation of the characteristic behavioral and electrophysiological patterns. For the 1AD determination, amplitude and frequency analyses were utilized. Amplitude analysis was performed by means of the Spike2 software. For frequency analysis the LFP signal was processed into power frequency spectrograms by the Matlab6.5 software (Fig 1) ${ }^{11-12}$.

Due to the number of animals and non-normal distribution of data, non-parametric Mann-Whitney-U-test was used at significance levels of $95 \%$ and $99 \%$. For statistical analysis the JMS software (SAS Software Corporation, Cambridge, England) was utilized.

\section{RESULTS}

The kindling process was divided into seven phases in order to be studied in details (Table 2). The first kindling session of each rat was named DO (first phase). The individual number of kindling sessions needed to the appearance of the first generalized behavioral seizure was divided into four quarters. The session referred to the first quarter was named D1, D2 to the second quarter, D3 to the third quarter, D4 for the fourth quarter (the session when the first generalized behavioral seizure appeared). D5 correspons to the fifth generalized behavioral seizure and D6 to the end of the kindling process (fully kindled state).

\section{Behavioral response}

During the hippocampal kindling process, each rat presented the first generalized behavioral seizure after a mean of 22.6 sessions ( $S D=9.2$ ). Eight animals completed the kindling process after a mean of 42.4 sessions ( $S D=8.8$ ). One rat showed a connector problem after D5. The duration of the behavioral response of each seizure (Racine score of three or more) was analyzed on D4, D5 and D6 phases. A statistically significant difference was observed between D4 and D6 $(p<0.05)$, presenting D6 a longer duration (Table 3).

The WDS amount of each session was quantified and a progressive decrease along the kindling process was observed. As the behavioral manifestation reached a higher Racine score, the number of WDS rate decreased, establishing an inverse correlation between the WDS occurrence and the development of the Racine motor stages (Table 4). A statistically significant fall on the number of WDS after the first generalized behavioral seizure (D4) was noticed $(p<0.05)$.

\section{Amplitude and frequency content}

Through the visual LFP inspection before (control period) and after the triggering stimulation, it was noted that 1AD consisted of waves of higher frequency and amplitude. After 1AD a relatively silent period was visualized, in which frequency and amplitude were inferior to the control period. The 2AD period (rebound) was marked by slow and high amplitude waves, which ceased simultaneously in all the eight recording channels, finalizing the kin- 
Table 4. Relationship between WDS and kindling phases.

\begin{tabular}{|c|c|c|c|c|c|c|c|}
\hline & DO & D1 & D2 & D3 & D4 & D5 & D6 \\
\hline Rat 1 & 8 & 13 & 7 & 9 & 3 & 0 & 0 \\
\hline Rat 2 & 21 & 13 & 12 & 11 & 1 & 0 & 0 \\
\hline Rat 3 & 1 & 8 & 3 & 1 & 1 & 0 & 0 \\
\hline Rat 4 & 7 & 5 & 11 & 2 & 0 & 0 & 0 \\
\hline Rat 5 & 2 & 7 & 3 & 4 & 3 & 1 & 0 \\
\hline Rat 6 & 15 & 7 & 2 & 6 & 4 & 0 & 0 \\
\hline Rat 7 & 8 & 6 & 2 & 4 & 0 & 0 & 0 \\
\hline Rat 8 & 18 & 20 & 16 & 15 & 0 & 0 & 0 \\
\hline Rat 9 & 10 & 4 & 6 & 8 & 0 & 0 & 0 \\
\hline Mean & 10 & 9.2 & 6.9 & 6.7 & 1.3 & 0.1 & 0 \\
\hline SD & 6.8 & 5.1 & 5.1 & 4.5 & 1.6 & 0.3 & 0 \\
\hline
\end{tabular}

An inverse relationship between WDS and epileptic behavioral was observed. There was an accentuated WDS fall with the appearance of the first generalized behavioral seizure (after $\mathrm{D} 4, \mathrm{p}<0.01$ ).

dling sessions. After D4 (generalized behavioral seizure) an increase on $1 A D$ complexity was observed and the visual differentiation between $1 A D$, interval and 2AD became difficult. Nevertheless, the after discharge end remained clear on all channels.

The LFP analysis coupled to the power frequency spectrograms showed high frequency discharges above $100 \mathrm{~Hz}$ (including ripples). Ripples (high frequency oscillations between 100 and $250 \mathrm{~Hz}$ ) were visualized superimposed to slower waves and were present on both hippocampi even before the behavioral seizures appearance (D4) (Fig 2).

\section{IAD duration analysis}

The LFP frequency and amplitude increase were used to determine 1AD duration on both hippocampi. 1AD start was marked when the frequency after the stimulus overcame the control period frequency and the end was marked when it returned to the control period value. The same procedure was used for amplitude analysis. Duration measurements were performed on signal recorded from posterior electrodes situated on both dorsal hippocampi.

A significant duration increase after the first behavioral seizure was observed in the right hippocampus (RH) through the frequency analysis, being D0 shorter than D4, $D 5$ and $D 6(p<0.05, p<0.01$ and $p<0.05$ respectively). D1 was shorter than D5 and D6 ( $<<0.05$ and $p<0.01)$, as well as $D 2$ was shorter than D4 and D5 $(p<0.05)$. The same recordings submitted to frequency analysis were used for amplitude analysis. D0, D1, D2 and D3 1AD duration were individually shorter than $D 5$ and $D 6$ ( $p<0.05$ and $p<0.01)$. In summary, a statistically significant $1 A D$ duration increase was observed in the right hippocampus (RH) after behavioral seizures appearance.

Regarding to frequency analysis performed on the

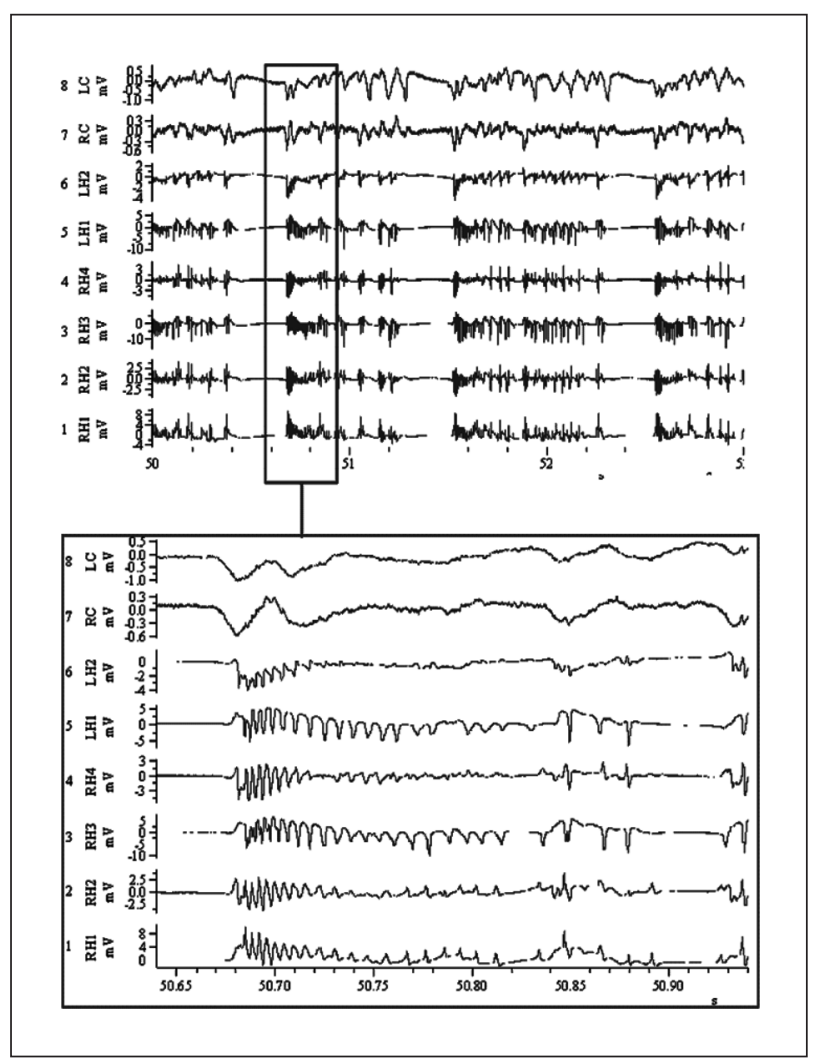

Fig 2. LFP signal evidencing high frequency oscillations (ripples) in both hippocampi. A period between the $50^{\text {th }}$ and the $57^{\text {th }}$ recording seconds was amplified to better demonstrate the ripples, which were found superimposed to slower waves. $\mathrm{RH} 1$ to $\mathrm{RH} 4$ channels recorded from the right hippocampus, $\mathrm{LH} 1$ and $\mathrm{LH} 2$ from the left hippocampus, $R C$ from the right frontal neocortex frontal and LC from the left frontal neocortex. Source: Experiment data.

signal recorded from the left hippocampus (LH), there was a statistically significant $1 A D$ duration increase when comparing D0 and D6 $(p<0.05)$. In amplitude analysis it 


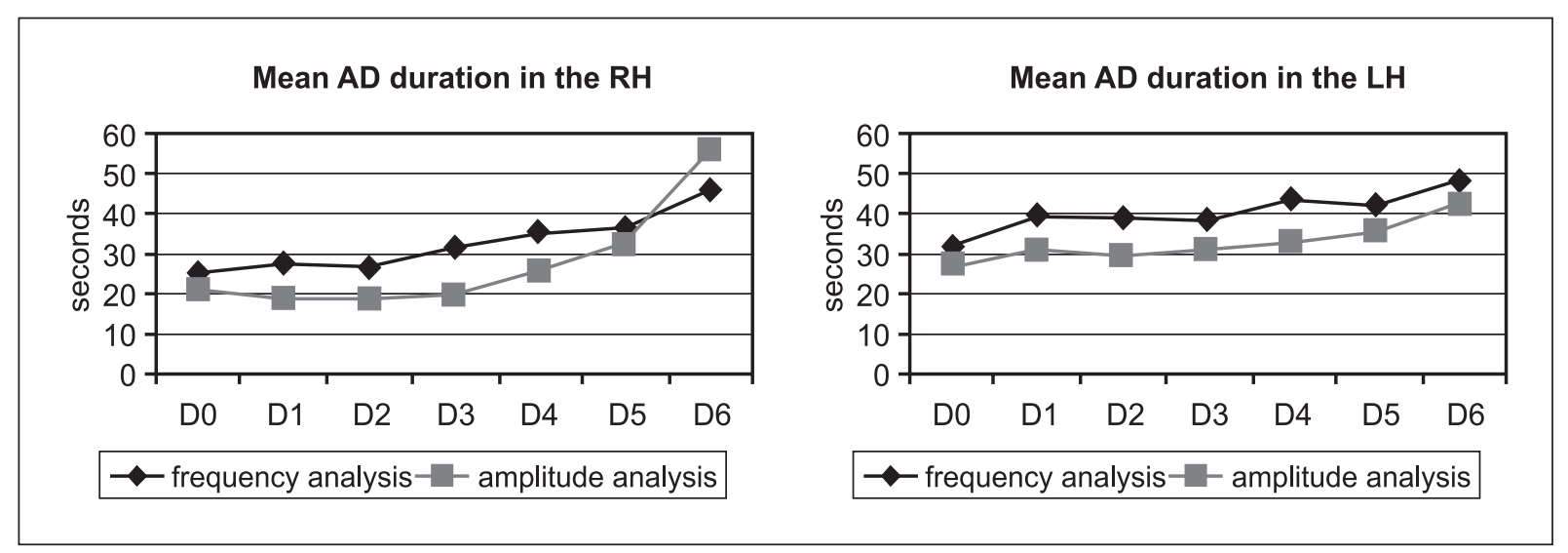

Fig 3. Hippocampal TAD duration (frequency vs. amplitude analyses). Comparison between frequency and amplitude analyses to the $1 A D$ duration measurement. Both methods presented congruent findings, revealing the progressive $1 A D$ duration increase regarding to both hippocampi with the kindling process. Source: Experiment data.

was observed that D0 was shorter than D4, D5 and D6 $(p<0.05, p<0.05$ and $p<0.01)$. D1, D2 and D3 were shorter than $p<0.01$. Thus, a statistically significant and progressive increase on $1 A D$ duration was also observed in the left hippocampus during the kindling process.

For an evolutional view of the duration increase, the means of each session are exposed (Fig 3).

By comparing both analytical tools, no significant difference in the 1AD duration between amplitude and frequency analysis in the LH was detected. Nevertheless, in the RH D1, D2 and D3 duration was shorter by amplitude analysis. $(p<0.01, p<0.05$ and $p<0.01)$.

\section{DISCUSSION}

Kindling phenomenon was first described in 1967 and since then has been extensively studied in animal models of temporal lobe epilepsy ${ }^{6}$. The phenomenon is characterized by a progressive installation through electrical stimulation repetition. Stimulation is delivered through deep brain electrodes and the obtained response is an afterdischarge (AD). The typical hippocampal AD seen in the initial kindling sessions is characterized by a low frequency basal pattern and has few behavioral correspondents. Ictal and behavioral events increase in complexity during the process, culminating with the appearance of high frequency oscillations and complex motor responses. Progressive development of electrophysiological and behavioral epileptic patterns are the features that mainly characterize the kindling phenomenon ${ }^{7,15,16}$. Kindling evolution may be seen in different cerebral areas, being more dramatically observed in temporal lobe structures, as the hippocampus and amygdala, as well as its adjacent cortical structures (perirhinal, entorhinal and piriform areas) ${ }^{17}$. Despite local stimulus application, hippocampal kindling constitutes a temporal lobe epilepsy model resulting in partial complex seizures with secondary generalization. This occurs due to the progressive neuronal recruitment from session repetition ${ }^{18,19}$. In very advanced phases, behavioral seizures may appear spontaneously, without the need of a triggering stimulus ${ }^{20,21}$.

Generalized seizures induced by hippocampal kindling, regardless of dorsal or ventral stimulation, present similar behavioral pattern when compared to those elicited through amygdala kindling. The AD pattern is, however, peculiar to the hippocampus. A typical hippocampal kindling session initiates with triggering stimulus followed by the $1 A D$ period, a relatively silent period, and the $2 A D$ period (or rebound period). This electrophysiologic pattern is typical of hippocampal kindling and is not seen in perirhinal cortex stimulation and amygdala stimulation ${ }^{16,22-24}$. This feature allows an electrophysiological evaluation of the electrode position even before histological analysis.

\section{Behavioral pattern}

In the amygdala kindling the Racine motor convulsive conditions are usually observed following the 1, 2, 3, 4, 5 sequence. The rats in this experiment skipped the first 2 stages, presenting a 3, 4, 5 sequence. This finding goes along with other reports about hippocampal kindling. Different from amygdala kindling, the hippocampal features involve a faster progression to more advanced motor stages, leading to a fast ictal generalization affecting the motor area. The progressive duration increase of behavioral seizures and the presence of the stages 6,7 and 8 (Pinel and Rovner Scale) are in conformance with previous descriptions that report a progressive complexity increase in both electrophysiological and behavioral features found in hippocampal kindling models ${ }^{22,23}$.

WDS is a stereotyped motor phenomenon which is associated with a characteristic high frequency generalized 
discharge. WDS may be found during the 1AD, 2AD or during the interval between them. The WDS rate per session initially increased and then decreased along the process, being an inverse relationship to the Racine motor convulsive states observed, which is in agreement with other experiments ${ }^{25-27}$.

\section{Electrophysiologic pattern}

All animals presented in every first session the typical hippocampal kindling electrophysiologic pattern, which is characterized by early appearance of high amplitude primary after discharges, followed by a relatively silent period and rebound discharge with slow and high amplitude waves (secondary afterdischarges), as well as the presence of WDS. Another interesting finding was the type of AD propagation pattern. The AD triggered in the right hippocampus presented a very fast spread to the contralateral hippocampus with a millisecond difference. This fact may be attributed to the presence of inter-commissural hippocampal fibers which hold only one synapse along their traject ${ }^{28}$.

In addition to the characteristic electrophysiologic pattern, the progressive 1AD duration increase on the stimulated hippocampus is also congruent with other descriptions ${ }^{26,27,29}$. In this study, the progressive 1AD duration increase was observed on both hippocampi, regardless of the type of analysis applied (amplitude and frequency). These two analytic modalities showed to be valuable tools in the evaluation of the afterdischarge triggered by hippocampal stimulation. The distinction between the $1 A D$ end and the beginning of the interval period was enhanced by the use of these tools. The signal processing form used in frequency spectrograms was especially helpful to analyse the signal obtained from the sessions in advanced stage, where simple visual analysis would be extremely difficult to be interpreted as the discharge complexity increased.

In afterdischarge frequency content, high frequency (HF) oscillations were encountered (between 100 and 400 $\mathrm{Hz}$ ), with spatial distribution and wave format similar to those described by Bragin et al. in kainate injected rats (intrahippocampal) and invasive hippocampal recordings from patients with TLE. Bragin et al. described the occurrence of these HF oscillations (ripples) restricted to the injected hippocampus ${ }^{14}$. Ripples described in this study were not only present in the stimulated hippocampus, but also in the contralateral one $\mathrm{e}^{1,12}$.

It has been demonstrated along this experiment that dorsal hippocampal kindling in rats provides features favoring temporal epileptogenesis studies, due to its progressive installation and increasing $A D$ and behavioral complexity. Another favorable feature of this model is the ability to trigger behavioral seizures, thus facilitating alternative testing of interventional paradigms.
Computational advances as described above may benefit research in experimental epilepsy, as new softwares may introduce sophisticated methodologies which will bring up more refined data analysis in classical animal models, such as better understanding of the role of high frequency discharges in hippocampal epileptogenesis as described in this study ${ }^{11-13}$.

These methods may also be applied to signal analysis obtained from different subjects (animal or computational models), improving the investigation of the seizure dynamics.

ACKNOWLEDGMENTS - We thank Carola Haas, Monika Häffner, Martin Müller and Armin Brandt for the aid in the experiment. This paper is a partial requirement to Cordeiro JC to obtain the Master Degree in Surgery by the Federal University of Paraná under the advisement of Araújo JC supervision.

\section{REFERENCES}

1. Commission on classification and terminology of international league against epilepsy: proposal of revised classification of epilepsies and epileptic syndromes. Epilepsia 1989;30:389-399.

2. Watson C, Andermann F, Gloor P, et al. Anatomic basis of amygdaloid and hippocampal volume measurement by magnetic resonance imaging. Neurology 1992;42:1743-1750.

3. Semah F, Picot MC, Adam C, et al. Is the underlying cause of epilepsy a major prognostic factor for recurrence? Neurology 1998;51:1256-1262.

4. Volcy Gomez M. Mesial temporal lobe epilepsy: its physiopathology, clinical characteristics, treatment and prognosis. Rev Neurol 2004;38:663-667.

5. Morrell F, Whisler WW, Bleck TP. Multiple subpial transaction: a new approach to the surgical treatment of focal epilepsy (see comments). J Neurosurg 1989;70:231-239.

6. Goddard GV. Development of epileptic seizures through brain stimulation at low intensity. Nature 1967;214:1020-1021.

7. Goddard GV, McIntyre DC, Leech CK. A permanent change in brain function resulting from daily electrical stimulation. Exp Neurol 1969; 25:295-330.

8. Paxinos G, Watson C. The rat brain in stereotaxic coordinates. Elsevier Academic Press, San Diego $6^{\text {th }}$ Ed, 2007:55-63.

9. Racine RJ. Modification of seizure activity by electrical stimulation. II Motor seizure. Electroencephalogr Clin Neurophysiol 1972;32:281-294.

10. Pinel JP, Rovner LI. Experimental epileptogenesis: kindling-induced epilepsy in rats. Exp Neurol 1978;15(Suppl 58:2):S190-S202.

11. Capurro A, Aertsen A, Schulze-Bonhage A. Evolution of high frequency components in depth EEG recordings during early stages of hippocampal kindling in rats. Epilepsia 2007;48(Suppl 3):S1-S66.

12. Capurro A, Aertsen A, Cordeiro JG, Meier R, Haeffner M, Schulze-Bonhage A. Evolution of correlations and high frequency components in EEG recordings from rat kindling and kainate models of temporal lobe epilepsy. Poster at "3rd International Workshop on Seizure Prediction in Epilepsy", Freiburg, September 29-October 2nd, 2007:47.

13. Capurro A, Cordeiro JG, Cordeiro KK, Meier R, Schulze-Bonhage A, Aertsen A. High-frequency components and correlation dynamics in local field potentials during hippocampal kindling in rats. To be submitted.

14. Bragin A, Engel Jr J, Wilson CL, Fried I, Mathern GW. Hippocampal and entorhinal cortex high-frequency oscillations (100-500 hz) in human epileptic brain and in kainic acid-treated rats with chronic seizures. Epilepsia 1999;40:127-137.

15. McNamara J. Kindling: an animal model of complex partial epilepsy. Ann Neurol 1984;16:72-76. 
16. Racine RJ, Rose PA, Burnham MW. Afterdischarge thresholds and kindling rates in dorsal and ventral hippocampus and dentate gyrus. Can J Neurol Sci 1972;4:273-278.

17. McIntyre DC, Kelly ME, Dufresne C. Fast and slow amygdala kindling rat strains: comparison of amygdala, hippocampal, piriform and perirhinal cortex kindling. Epilepsy Res 1999;35:197-209.

18. McIntyre DC. Differential amnestic effect of cortical vs. amygdaloid elicited convulsions in rats. Physiol Behav 1970;5:747-753.

19. McIntyre DC. Effects of focal vs generalized kindled convulsions from anterior neocortex or amygdala on CER acquisition in rats. Physiol Behav 1979;23:855-859.

20. Pinel JPJ, Mucha RF, Philips AG. Spontaneous seizures generated in rats by kindling: a preliminary report. Physiol Psychol 1975;3:127-129.

21. Wada JA, Osawa T. Spontaneous recurrent seizure state induced by daily electric amygdaloid stimulation in Senegalese baboons (Papio papio). Neurology 1976;26:273-286.

22. Burnham WM. Primary and "transfer" seizure development in the kindled rat. Can J Neurol Sci 1975;2:417-428.
23. Grace GM, Corcoran ME, Skelton RW. Kindling with stimulation of the dentate gyrus. I. Characterization of electrographic and behavioral events. Brain Res 1990;509:249-256.

24. Leung LW. Hippocampal electrical activity following local tetanization. I. Afterdischarges. Brain Res 1987;419:173-187.

25. Leung LS, Shen B. Hippocampal partial kindling decreased hippocampal $\mathrm{GABA}_{\mathrm{B}}$ receptor efficacy and wet dog shakes in rats. Behav Brain Res 2006;173:274-281.

26. McIntyre DC, Kelly ME. Are differences in dorsal hippocampal kindling related to amygdala-piriform area excitability? Epilepsy Res 1993;14:49-61.

27. Lerner-Natoli M, Hashizume A, Rondouin G, Baldy-Moulinier M. Wetdog shaking behavior in rats in hippocampal kindling. CR Seances Soc Biol Fil 1983;177:93-101.

28. Paxinos G. The rat nervous system. San Diego: Academic Press $2{ }^{\text {nd }} E d$, 2004:443-486.

29. Leung LS, Zhao D, Shen B. Long-lasting effects of partial hippocampal kindling on hippocampal physiology and function. Hippocampus 1994;4:696-704. 